\title{
Application of laser image technology for deformation monitor of building
}

\author{
WEI Li \\ Department of Mechanical \& Electrical Enginerring, Tangshan College, Hebei Tangshan, \\ 063000, China \\ ysuweili@163.com
}

Keywords: virtual instrument; laser image; deformation monitoring; microcontroller; wireless transmission

\begin{abstract}
According to the actual demand of deformation monitoring for large buildings, the technology of laser image based on virtual instrument is put forward. The location of laser light spot relative to the reference position and the variation of tilt angle are measured, which can calculate the vertical deformation value of displacement. In the measurement process, it uses the function library of image processing in LabVIEW to perform the read and processing of image, which has the advantages of convenient operation and accuracy location of measurement spot center In order to satisfy the requirement of on-line monitoring, the measurement data is transmitted to the host computer for operation and display using the way of wireless. The measurement data can be read in real time and can also be saved for the inquiry.
\end{abstract}

\section{Introduction}

In order to detect the performance of buildings, bridges, dams and other large buildings, it usually needs to determine the deformation of key parts for these buildings. The deformation is caused by changes of natural conditions and long-term load even overload. It maybe bring damage when exceeds the certain threshold. At present, the direct measurement method of the displacement sensor and the indirect measurement method of strain measurement are used to measure the deformation. For example, it can measure position change of the key part relative to some benchmark by displacement sensor. Consequently, the displacement of whole structure can get. But the sensor must be perpendicular to the deformation position of measured object and must be located in base position close to target, which is the biggest problem. In addition, it consume a lot of manpower and material resources to establish the base position, sometimes even impossible to perform when measuring deformation of bridge across the river. The primal problem of indirect measurement method is that it uses some hypothesis of dynamics theory in deformation calculation, but sometimes the actual object is not entirely consistent with these hypotheses, which cause larger measurement error. Sometimes it is not used to measure the deformation of the object when not know well the geometrical dimensions and material properties of the measured object.

In recent years, there are some new methods of deformation monitoring with the development of computer technology, sensor technology and network technology, such as tension line method[1], communication tube method[2], tilt sensor method[3], GPS method[4], networking method[5]. Moreover, the method of laser image has the advantages of convenient installation, low maintenance cost and high precision, which becomes research hotspot[6]. In this paper, the virtual instrument technology is applied to the laser image method. Compared with other methods, this method has the advantages of simple algorithm, high accuracy, easy installation, etc..

\section{System Structure}

The system is composed of laser emitter, laser receiver and the upper computer. The laser beam should be long-term stability, anti-interference and high positioning accuracy, in order to ensure the accurate positioning of the spot center[7]. A diode pump solid state laser(DPSSL) with reliable 
stability and limberness of light spot shape is used in the study. Laser emitter consists of laser controller, laser power and laser collimator. Laser receiver consists of semi-transparent target, laser lens, image sensor and position sensor. The quality of light spot determines the measurement accuracy of the system. The semitransparent laser target is connected with the lens hood to ensure uniform spot, concentrated center energy and stable position. The signals from image sensor and obliquity sensor are transmitted to microprocessor. Then the processed signal is transmitted to the host computer for remote monitoring by wireless mode. The system structure diagram is shown in fig. 1.

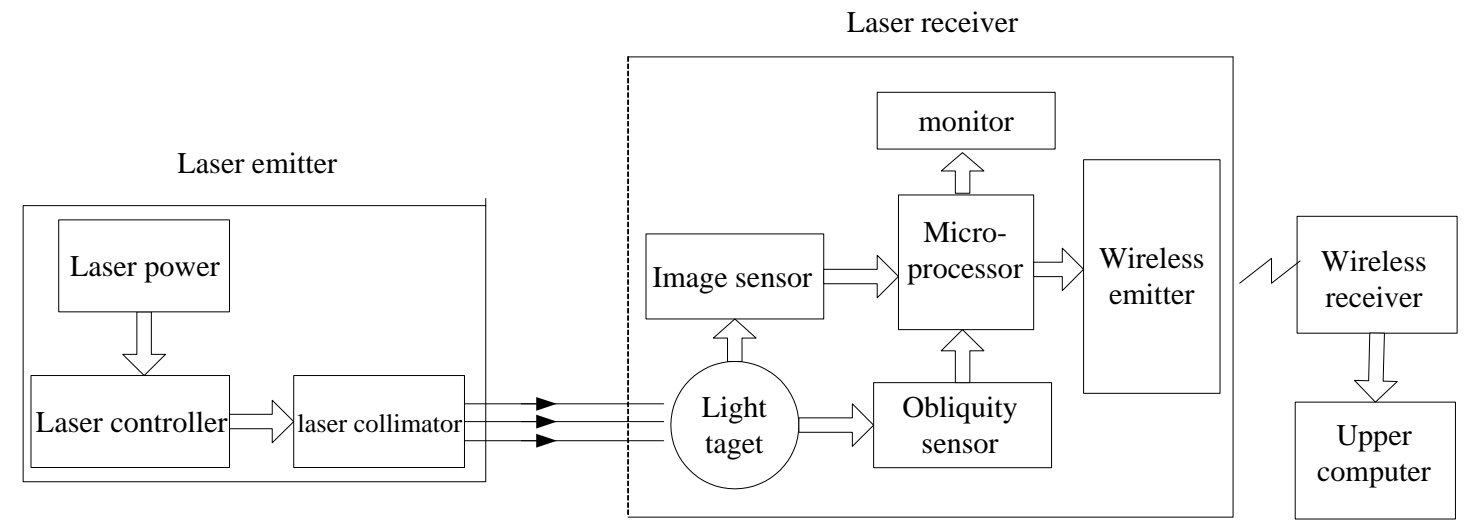

Fig.1 System block diagram

\section{Measurement principle of system}

The laser emitter is installed on the measurement base point of buildings. The laser is used as an extension of the measurement datum. Because the laser has good linearity and stability, it can ensure the accuracy and reliability of the measurement. Laser receiver is installed at the measured point, and the change of the spot position on the laser target reflects the deformation of the measured point relative to the measurement datum. In addition, the laser spot receiver will tilt relative to the datum, which caused by the deformation. The illustrative diagram is shown in fig.2.

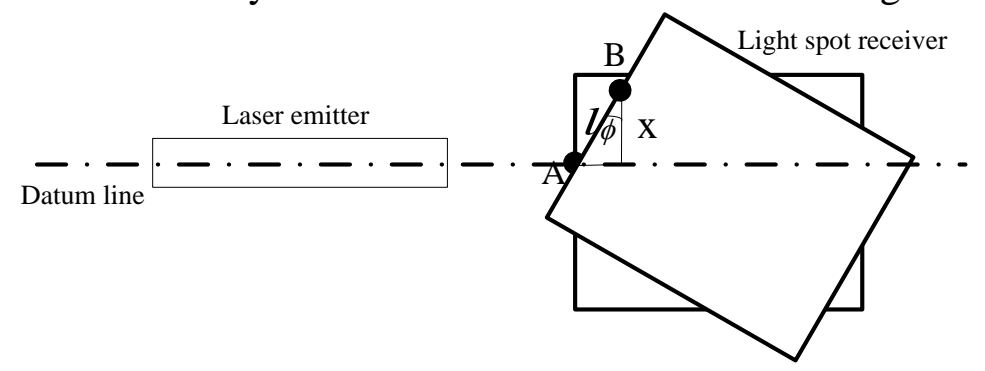

Fig.2 Principle figure of deformation measurement

As shown in the fig.2, it designates that $\mathrm{A}$ is the datum position, $\mathrm{B}$ is the laser light spot position, $l$ is the length of $\mathrm{AB}$ line, which is the deformation relative to datum plane. $\phi$ is the obliquity of light sot receiver relative to datum plne. $\Delta$ is the displacement value of the measured point perpendicular to the datum plane, so $\Delta$ is the deformation result. The relationship between the three is shown in the eq.(1). The vertical deformation of the position can be analyzed by collecting the center coordinates of the spot image.

$\Delta=l \cos \phi$ 


\section{Laser image data processing algorithm}

The USB HD image sensor is used to collect the data of the laser spot to the computer. Currently popular image processing platform includes OpenCV, MATLAB and $\mathrm{C}$ or $\mathrm{C}++$ is the main programming language. These methods require that the developers must be familiar with all kinds of image processing algorithms. In the design of image processing system, it uses LabVIEW of NI Company from the United States. Vision Development Module is the development toolkit of LabVIEW, which includes the utility software Vision Assistant and image processing VI library IMAQ Vision. Vision IMAQ is a powerful function library, including histogram analysis, query table transform, Walsh transform, filtering, morphological frequency domain analysis and other image processing VI[8]. The main advantage is that it includes the various classical algorithms and programming efficiency is higher than the text based development environment. It is easy to integrate with the collection hardware and software.

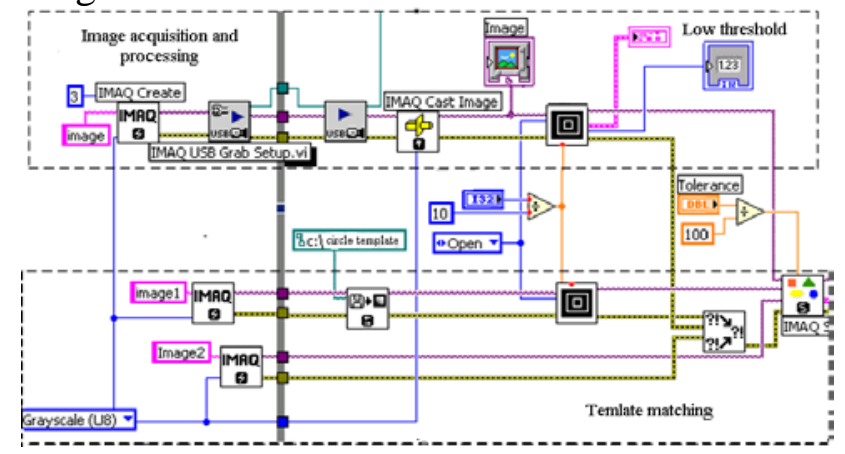

Fig.3 Light spot reception program

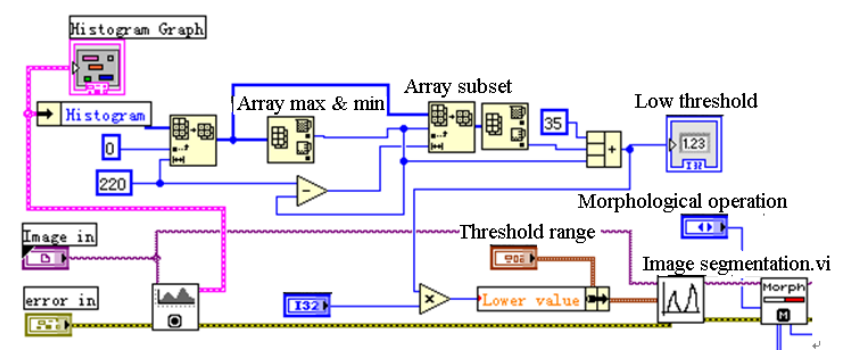

Fig.4 Light spot image processing program

The spot receiving program is shown in Fig.3. It includes image acquisition and processing and template matching parts. First it creates the image and outputs the reference handle of the image, then calls the USB IMAQ function to initialize and read USB camera device. This function transmits the image from image sensor to the computer. The function of Cast Image IMAQ converts spot image to 8 bit gray images. It analyzes the image after image acquisition, as shown in Fig.4. The gray histogram of the spot image can be get through IMAQ histogram function. According to the character of spot image, the gray value illustrates the target background and target spot parts. The parameter of Histogram can be parsed by the output value of histogram function. The data type is array, so the valley threshold of bimodal gray histogram can be counted through the array functions. It can use this boundary threshold between background and object to segment the image. For changing the granular structure of image and smoothing the pattern changes, It can use the function of VI IMAQ Gray Morphology in the grayscale morphological library after the image segmentation to perform the arithmetic of first expansion after corrosion [9].

Because of the interference of the ambient light, the shape of light spot is irregular and it can't get the coordinates of the center of the spot better. So a circular template is first made according to the size of the spot, as shown in Fig.5. The function of Shape IMAQ Match Tool is used to match the image after processing with the circle template image, so a regular circle shape can be get from the original light spot image. It is easy to get the coordinates of the circle. The value of $l$ in the eq.(1) can be obtained by measuring the position of the center of the spot.

According to the requirement of the measurement environment, the angle sensor LCA320T with small size, low power consumption and dual axis voltage output is selected. The sensor uses the non-contact measurement principle. The angle and the output voltage is linear and the output voltage is transmitted to the computer through two channels. As shown in Fig.6, the front panel shows the pattern of the measurement module, the gray histogram and the center coordinates and the dip angle of the spot. 


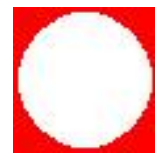

Fig.5 Circle template

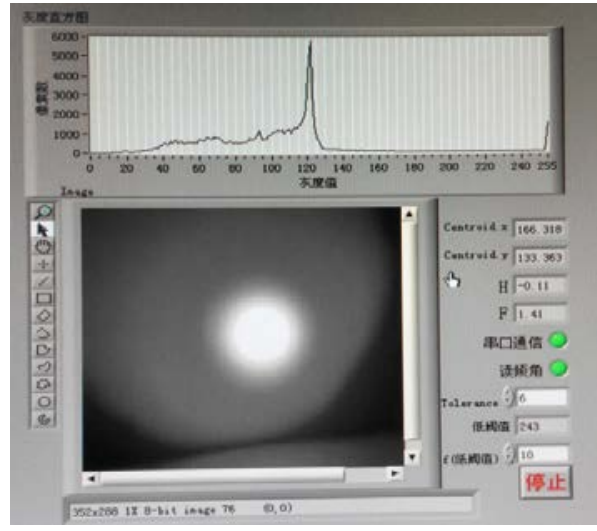

Fig.6 Light spot image and histogram

\section{System Implementation}

Generally the location of deformation monitoring point is high for general building and it may be not to install the monitoring device. So it adopts the embedded system to perform measure. First each functional modules of the system is connected as shown in Fig. 1 and the material object is shown in Fig.7. In order to facilitate the program from the LabVIEW development environment and LabVIEW running engine, the procedures of spot collection and dip measurement are converted into installation program in the development system. Then the installation program is written into SST49LF008A, which is a BIOS memory chip. In this way the program can be I/O operated directly with the sensors, network and other peripheral devices and it can be separated from the specific hardware operation. In order to read data conveniently, the wireless transmission is used to transmit data to the remote computer and the wireless receiving module uses the way of serial port to the USB port to connect the notebook.

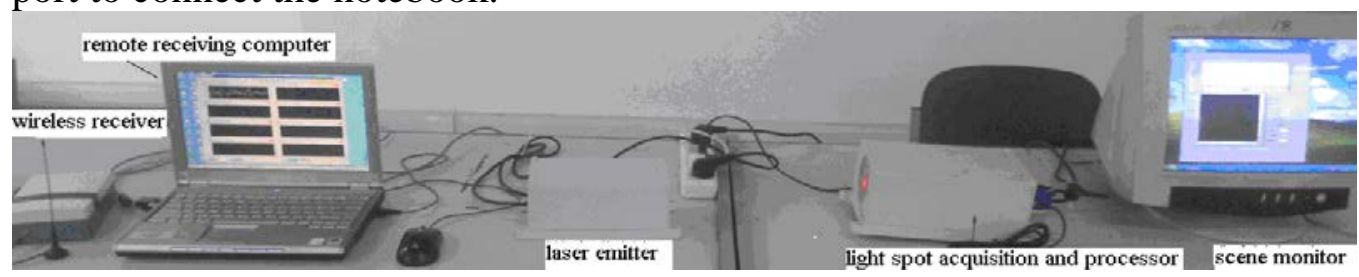

Fig.7 Physical objects connection chart

The system is tested after built in the laboratory. According to the requirements of the building deformation monitoring, it need to set multiple measurement devices in key points and use different channels for receiving acquisition data. The receiving program is shown in Fig.8. First, it need to configure the serial port, then read the serial character, that is the measured value of displacement and the angle. It uses the sub VI of "character index" to convert the character to numerical value and the cosine operation to output the data in the graphics control. The test procedure is only testing one measurement system for manual deformation operation and the upper computer displays the output results as shown in Fig.9. The measurement results can be recorded in real time and saved as EXCEL file. It can be played back and easy to analyze the data in the future.

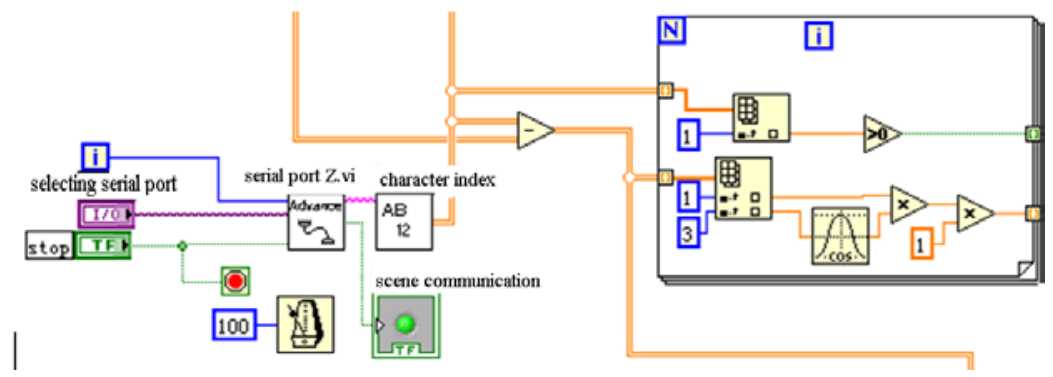

Fig.8 Remote receiving program 


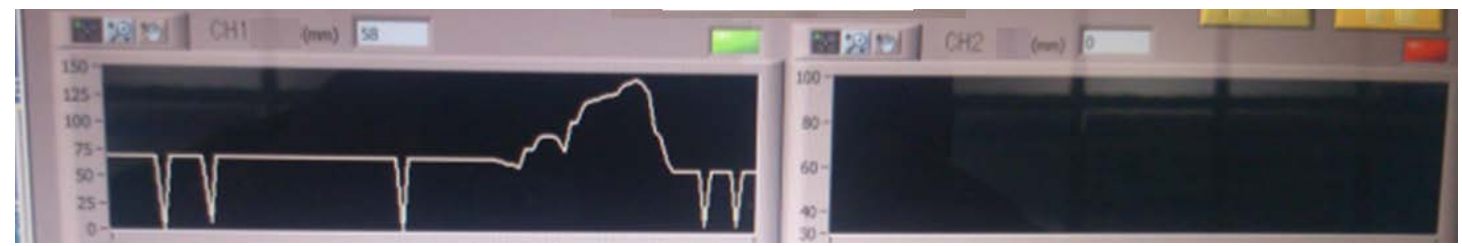

Fig.9 Receiving display interface

\section{Conclusion}

Deformation monitoring is important for the health monitoring of large buildings, which is hot research topic in recent years. With the development of laser technology and digital image processing technology, these technologies will be applied to large scale building deformation monitoring and will bring more in-depth research. The deformation results of the laser image are obtained by measuring the displacement and the dip angle based on the virtual instrument. In the process of the spot location, the image analytical functions in LabVIEW, such as gray histogram threshold analysis, image segmentation and morphological function are more convenient and ensure the accuracy of the center location measurement, remote transmission and real time measurement.

\section{Reference}

[1]Lan zhangli, Yang Xiaofan. Non-contact bridge def lection measurement system using weighted-stretched-wire [J]. Chinese Journal of Scientific Instrument, 2008,29(5): 1058-1062

[2]Zhu Shifeng, ZHOU Zhixiang, WU Haijun. Application of semi-closed connected pipe differential pressuresensor in bridge deflection measurement[J]. Transducer and Microsystem Technologies, 2014, 33(1): 150-153.

[3]Li Hongwei, Zeng guoliang, Hou liwun. A practical method for measuring the deflection of bridge by using the angle sensor[J]. Journal of Disaster Prev ention and Mitig atio n Engineering, 2010,30(9): 275-278

[4]Yu Xianwen, Xue Honglin. Method of bridge vibration monitoring based on GPS and accelerometer [J]. Journal of Southeast University( Natural Science Edition), 2013,43(2): 329-333

[5]Shang Jinguang. Research on the tunnel deformation monitoring and forewarning system based on IOT[D]. Southwest Jiao tong University Master Degree Thesis.2009.

[6]Zhang Ben-niu, LI Xingxing , Song Jun, Yan Guo. Application Study on Laser Projection Sensor for Bridge Def lection Detection [J]. Chinese Journal of sensors and actuators,2009, 22(5): 755-759.

[7]Lan Zhang li, Yan Guo Yang xiao fan.Location Algorithm of the Laser Spot Center Based on Dynamic Gradient[J].Computer science, 2008, 35(8): 223-226.

[8]Lei zhenshan, Xiao chenyong, Weili. LabVIEW Advanced programming and application of Virtual Instrument Engineering [M]. Beijing: Chinese railway press house, 2013: 231-241.

[9] Ruan qiuqi. The basis of digital image processing [M]. Beijing: Tsinghua University press, 2009: 320-344 